\section{CODED BLOCK NEURAL NETWORK VLSI SYSTEM USING AN ADAPTIVE LEARNING-RATE TECHNIQUE TO TRAIN CHINESE CHARACTER PATTERNS}

\section{B. Y. Chen, M. W. Mao and J. B. Kuo}

Indexing terms: Neural networks, Pattern recognition

A coded block neural network VLSI system is presented that uses an adaptive learning-rate technique to train Chinese character patterns. Using the adaptive learning-rate technique, 500 Chinese characters have been successfully trained in $47.2 \mathrm{~h}$ using a 28 MIPs computer.

Introduction: A pattern recognition system composed of a translation-invariant network and a standard adaptive twolayer network is used to map a retinal image into multibit outputs [1-3]. The standard adaptive two-layer network [4, $5]$ can be trained to provide output responses corresponding to the original image as required. Software implementation of the adaptive neural network using back propagation [6-8] has been demonstrated. For large-volume Chinese character pattern recognition, training time is critically determined by the number of neurons and connections between them. A block adaptive network structure suitable for VLSI implementation has been reported to enhance the learning speed [9] for alphanumeric recognition. A coded block neural network system using the conventional back propagation algorithm suitable for VLSI implementation, which requires an order-ofmagnitude fewer local blocks, has also been suggested to provide a faster learning process for alphanumeric recognition [10]. However, for training large-volume patterns such as Chinese characters, the coded block neural network system using the conventional back propagation algorithm still needs a substantial learning time [11]. In this Letter, a coded block adaptive neural network system using an epoch-based back propagation algorithm with an adaptive learning rate for training large-volume Chinese character patterns, which is insensitive to the initial weights and irrelevant to the order of input patterns and suitable for VLSI implementation, is presented.

Adaptive epoch-based back propagation: Fig. 1 a shows a coded block [9] neural network system using the back propagation algorithm to train large-volume input patterns. Fig. $1 b$ shows a local block in the system. Owing to its isolated local block structure, the training time has been much shorter as com pared to a standard system using the conventional back propagation algorithm [10]. However, for large-volume pattern recognition applications, the conventional back propagation algorithm cannot provide an efficient training process. To facilitate training of large-volume patterns, an improved back propagation algorithm, 'epoch-based' back propagation, has been developed. Instead of updating each individual weight value immediately after each pattern is presented at the input, the new epoch-based back propagation algorithm updates each individual weight value in the first and second layers only after all patterns have been sequentially applied in an epoch. In the $j$ th local block in the epoch-based system as shown in Fig. 1b, the weight value associated with the synapse between the local block output and the $k$ th neuron in the first

\section{Table 1 IMPORTANT EQUATIONS}

layer is changed only once per epoch as shown in eqn. 1 in Table 1. Initially, a learning rate equal to the inverse of number of the input patterns is selected. After every training epoch, the learning rate associated with a specific pattern
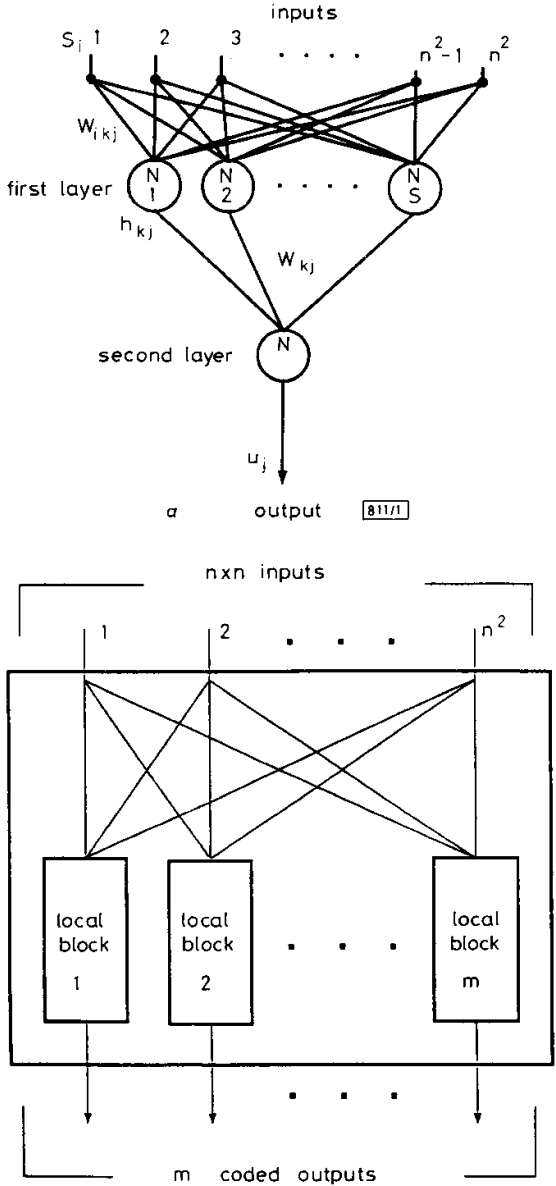

$b$

$8 i \sin$

Fig. 1 Coded block adaptive neural network structure and simplified hierarchical structure of local block

a Adaptive neural network structure

$b$ Simplified hierarchical structure of local block

stays unchanged if the output of the system for the specific pattern matches its desired output. Otherwise, the learning rate increases by a value equal to $20 \%$ of its initial value. After the specific pattern matches its desired output, the learning rate associated with that specific pattern is decreased by a value equal to $20 \%$ of its initial value. If further training is necessary such that the learning rate associated with the specific pattern is supposed to be lower than its initial value, the

Eqn.

\begin{tabular}{|c|c|c|c|}
\hline & $\Delta w_{k j}(n)=\sum_{i=1}^{p} \eta^{(l)}(n) \delta_{j}^{(l)}(n) h_{k j}^{(d)}(n)+\alpha \Delta w_{k j}(n-1)$ & $k=1,2, \ldots, s$ & \\
\hline & $\Delta w_{i k j}(n)=\sum_{i=1}^{p} \eta^{(i)}(n) \delta_{k j}^{(l)}(n) s_{i}^{(l)}+\alpha \Delta w_{i k j}(n-1)$ & $k=1,2, \ldots, s$ & $i=1,2, \ldots, n^{2}$ \\
\hline & $\delta_{k j}^{(l)}(n)=h_{j k}^{(l)}(n)\left(1-h_{k j}^{(l)}(n)\right) \delta_{j}^{(l)}(n) w_{k j}^{(l)}(n-1)$ & $k=1,2, \ldots, s$ & \\
\hline & 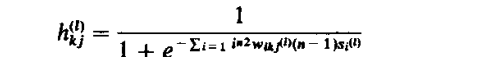 & $k=1,2, \ldots, s$ & \\
\hline
\end{tabular}

ELECTRONICS LETTERS 8th October 1992 Vol. 28 No. 21 
learning rate has been set to be at its initial value. After training is successfully concluded, the learning rate associated with each input pattern may be different. $A$ value of 0.85 for the momentum $\alpha$ has been used. For the epoch-based system, the weight value associated with the synapse between the ith inpu $\left(s_{i}\right)$ and the $k$ th first layer neuron is updated only once per epoch according to the formula as shown in eqns. $2-4$ in Table 1.

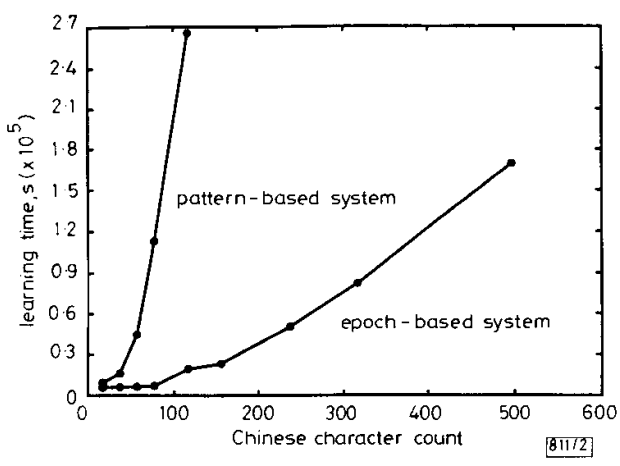

Fig. 2 Learning time against input pattern numbers for training largevolume Chinese characters using coded block neural network system with epoch-based and pattern-based back propagation algorithms

Comparison with simulation results and discussion: To confirm the advantages of the epoch-based back propagation algorithm with an adaptive learning rate in the coded block neural network system for training large-volume patterns, performance of the system in terms of learning time for training 500 frequently-used Chinese characters composed of $24 \times 24$ lattice dots has been successfully obtained. In the coded block structure under study, there are 16 local blocks with 12 neurons in the first layer in each local block. Fig. 2 shows the learning time against the number of Chinese characters used as input patterns. The learning time is measured in terms of CPU time using a 28 MIPs computer. For a small volume of input patterns, the difference in training time between the systems using pattern-based and epoch-based back propagation algorithms is small. However, as the number of input patterns increases, the difference in learning time between the two algorithms grows wider. For training 500 patterns, the learning time for the system using the coded block structure with pattern-based back propagation algorithm is just not practical on a 28 MIPs computer. On the other hand, with the epoch-based algorithm, the coded block system can learn 500 input patterns in $47.2 \mathrm{~h}$. Fig. 3 shows the learning time against the learning rate for a coded block system using the patternbased and the epoch-based back propagation algorithms for training 40 Chinese characters. The initial learning rate of the

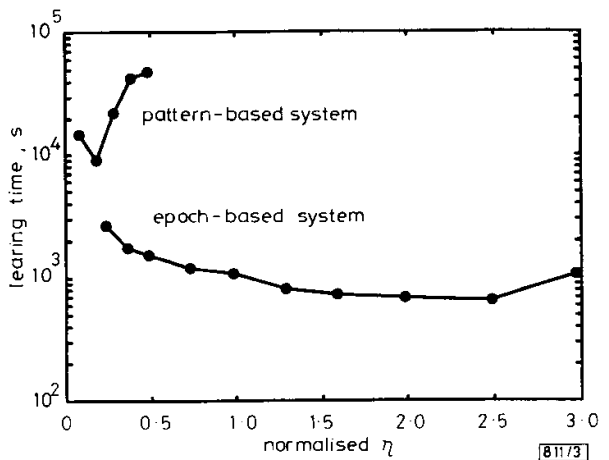

Fig. 3 Learning time against initial learning rate for training 40 Chinese characters using coded block neural network system with epochbased and pattern-based back propagation algorithms

Learning rate is normalised by a value equal to the inverse of the number of input patterns epoch-based system is normalised by a value equal to the inverse of the number of input patterns. Using the patternbased system, the system can have an acceptable learning time only for a limited range of learning rate. On the other hand, using the epoch-based back propagation algorithm, the learning time stays relatively insensitive to the initial learning rate. This is important for the system with an adaptive learning rate technique because the learning rate can have more space to be adjusted automatically. For training up to 500 input patterns, using the epoch-based back propagation algorithm with the adaptive learning rate technique, a much quicker convergence is always guaranteed.

23rd July 1992

B. Y. Chen, M. W. Mao and J. B. Kuo (Rm. 526, Dept. of Electrical Eng., National Taiwan University, Roosevelt Rd. Sec. 4, \# I, Taipel 106-17, Taiwan)

\section{References}

1 WWrow, B., WINTER, R. G., and BAXTER, R. A.: 'Layered neural nets for pattern recognition', IEEE Trans., 1988, ASSP-15, pp. 1109 1117

2 RUMELHART, D. E, and MCCLELLAND, J.: 'Parallel distribution processing' (Massachusetts Institute of Technology, 1986)

3 'DARPA Neural Network Study', October 1987-February 1988

4 MIRCHANDANI, G., and CAO, W.: 'On hidden nodes for neural nets', IEEE Trans., 1989, CAS-16

5 TOURETzKy, D. S., and pomerlead, D. A.: 'What's hidden in the hidden layers', Neural Networks, 1989

6 JONES, w. P., and HOSKINS, J.: 'Back-propagation: a generalized delta learning rule', Byte, pp. 155-162

7 YAMADA, K., KAMI, H., TSUKUMO, J., and TEMMA, T.: 'Handwritten numerical recognition by multi-layered neural network with improved learning algorithm'. Int. Joint Conf. of Neural Networks, 1989

8 VOGL, T. P., MANGiS, J. K., and RiGLER, A. K.: 'Accelerating the convergence of the back propagation method', Biol. Cybern., 1988 pp. $129-263$

9 kuo, J. B. et al.: 'A coded block adaptive block neural network structure for pattern recognition VLSI'. Dig. 1991 Int. Symp. on VLSI Tech. Sys. and Applications

$10 \mathrm{KUO}$, J. B., and MAO, W. C.: Adaptive neural network structure with coded local blocks for pattern recognition VLSI'. Proc. 1991 IJCNN

11 MAO, M. W., and Kuo, J. B.: 'A coded block adaptive neural network system with a radical-partitioned structure for large-volume Chinese character recognition', Neural Networks, 1992

\section{ULTRALOW ERROR RATE AND DISPERSION-FREE TRANSMISSION OF 2.5 Gbit/s SONET FOR GIGABIT NETWORKS}

H. Izadpanah, A. Elrefaie, Chinlon Lin and J. L. Gimlett

Indexing terms: Optical communication, Optical fibres, Optical dispersion

It is demonstrated that for an NRZ modulated $1310 \mathrm{~nm}$ optical transmitter, dispersion-free transmission can be achieved up to $\sim 250 \mathrm{~km}$ using dispersion-shifted singlemode fibre (DS-SMF) and optical pulse compression. This result fibre (DS-SMF) and optical pulse compression. This result
implies that DS-SMFs have a potential advantage over stanimplies that DS-SMFs have a potential advantage over stan-
dard SMFs for dual-window $(1310 / 1550 \mathrm{~nm})$ multigigabit per dard SMFs for dual-window $(1310 / 1550 \mathrm{~nm})$ multigigabit per performance of experimental prototype $2.5 \mathrm{Gbit} / \mathrm{s}$ SONET optical transmission systems are presented with measured error rates smaller than $10^{-15}$.

Introduction: The need to transport services such as supercomputer data with gigabit per second access rates is emerging rapidly. Self-healing, high-speed SONET/ATM ring networks are capable of transporting data packets at gigabit per second rates [1]. In such networks, fibre chromatic dispersion is a chief source of error-rate penalties, particularly in dual-window $1310 / 1550 \mathrm{~nm}$ wavelength networks with path lengths greater than $40-60 \mathrm{~km}$. High-speed transmission

ELECTRONICS LETTERS 8th October 1992 Vol. 28 No. 21 